\title{
Which positive predictive value for Diffusion-Weighted Magnetic Resonance Imaging for children's cholesteatomas? A retrospective study of 126 MRI exams
}

\author{
Rémi HERVOCHON ${ }^{1}$, MONIQUE ELMALEH-BERGES ${ }^{2}$, Martine François ${ }^{3}$, ALIX \\ MARHIC $^{4}$, ABDULLAH BAHAKIM $^{2}$, Natacha Teissier ${ }^{3}$, and Thierry Van Den Abbeele ${ }^{3}$ \\ ${ }^{1}$ University Hospital Pitié Salpêtrière \\ ${ }^{2}$ Robert-Debré Mother-Child University Hospital \\ ${ }^{3}$ Hopital Universitaire Robert-Debre \\ ${ }^{4} \mathrm{APHP}$
}

April 28, 2020

Which positive predictive value for Diffusion-Weighted Magnetic Resonance Imaging for children's cholesteatomas? A retrospective study of 126 MRI exams

Word count : 1454

All authors have not any conflict of interest

No financial Support

Legal representative of all patients gave their informed consent.

Presentation at IFOS congress in Paris: June $27^{\text {th }}, 2017$

\section{KEY POINTS}

The aims of this study were first to calculate the Positive Predictive Value (PPV) of DW-MRI to detect cholesteatoma and then to analyze false positives.

- The number of DW-MRI for identification of cholesteatoma has grown in ten years

- We calculated an $89 \%$ PPV

- DW-MRI sensitivities were $100.0 \%$ and $70.7 \%$ for respectively keratin and squamous epithelium.

- DW-MRI hypersignal is not synonymous of cholesteatoma diagnosis. Indeed, this diagnosis relies on the importance of a proper otoscopic examination, a suggestive medical history, CT scan data and analysis of other MRI sequences, including T1-weighted sequence, to rule out other etiologies of middle ear DW-MRI hypersignal.

\section{INTRODUCTION}

Diffusion-Weighted Magnetic Resonance Imaging (DW-MRI) has become in the past 10 years a key element in the diagnostic management of cholesteatoma, when otoscopy and CT are inconclusive in surgery-naive patients or to assess post-operative recurrence or residual cholesteatoma. However, not all DW-MRI hypersignals are cholesteatoma. The aims of this study were first to calculate in our pediatric series the Positive Predictive Value (PPV) of MRI with DWI sequences and then to analyze false positives.

MATERIALS AND METHODS 


\section{Ethical considerations}

Legal representative of all patients gave their informed consent.

All temporal bone MRI with DWI sequences performed in our pediatric university hospital between 2005 and 2015 were included retrospectively. All patients with a cholesteatoma diagnosis on the MRI report and who underwent surgery were included. All scans have been analyzed by a pediatric ENT specialized radiologist (MEB). For each patient, intraoperative findings and histological findings on the surgical report and on the pathological report were collected. The PPV was established. False positives were analyzed and compared to true positives. The chi-square test was used to compare binary qualitative variables, and the Student test was used to compare continuous quantitative variables. An $\alpha$ risk $<0.05$ was tolerated. Statistical analysis was performed on R software.

Imaging technique: all examinations were carried out on a 1.5T MRI scanner (INTERA; Philips Medical Systems; Best, The Netherland) using a head coil or a paired surface coil. All DW-MRI included a coronal T2 turbo-spin echo (TSE) sequence with $2 \mathrm{~mm}$ thick slices, an axial 3D T2 TSE high resolution sequence (DRIVE), an axial T1 TSE sequence $2 \mathrm{~mm}$-thick slices sequence and two DWI sequences axial and coronal. DWI sequences were DWI-TSE sequences, and apparent diffusion coefficient (ADC) maps were calculated.

\section{RESULTS}

Over the period between 2005 and 2015, 126 petrous bone DW-MRI were performed in our pediatric hospital. Distribution of the number of exams across the years is shown on Figure 1.

Forty-six MRI records, which were considered positive for cholesteatoma, were included. The average age of patients undergoing the MRI scan was $14.2 \pm 5.3$ years old. The average delay between imaging and surgery was $111 \pm 78$ days. Out of the 46 positives DW-MRI, a cholesteatoma was found surgically in 41 patients, which represents an $89 \%$ PPV. Regarding the histological reports, out of these 41 surgeries, keratin was found in all cases, and squamous epithelium in 29 cases. Therefore, DW-MRI sensitivities were $100.0 \%$ and $70.7 \%$ for respectively keratin and squamous epithelium.

True and false positives were compared (Table 1). There was no statistically significant difference between false positives and true positives, especially in terms of age, number of prior surgery, time between MRI and surgery, and ossicular prosthesis presence. However, two trends appear. First, $20.0 \%$ of the false positives had never had any previous ear surgery, versus $7.3 \%$ in true positives $(\mathrm{p}=0.341)$. Second, the average size of the diffusion hypersignal was smaller in false positives than in true positives: $6.68 \pm 4.29 \mathrm{~mm}$ versus $9.34 \pm 4.46$ $\mathrm{mm}$, although this difference was not significant $(\mathrm{p}=0.251)$.

The MR images of the 5 false positives DW-MRI were reviewed, with both surgical and pathological reports:

- Case 1: a 14 years old girl with Turner syndrome. On the CT, the middle ear was filled with isodense content, showing neither convex limits nor ossicular lysis. On the MRI, there was a T1 and T2 hypersignal with DW hypersignal. Cholesterol granuloma was found.

- Case 2: a 16 years old girl. On CT, there was an antral and attical filling with a demineralization of the long process of the incus. On the MRI, there was a $4 \mathrm{~mm}$ DW-MRI hypersignal, with restricted diffusion and T1 hyposignal, consistent with an attical small cholesteatoma.

- Case 3: a 16 years old girl. On CT, middle ear cavities were opacified. An attical DW-MRI hypersignal, with restricted diffusion on ADC map, $6.5 \mathrm{~mm}$ large, was found with a slight T1 hypersignal: a cholesterol granuloma was confirmed surgically.

- Case 4: a 12 years old boy. Posterior attical cavity, antrum and mastoid were filled by T1 hyperintense material consistent with cholesterol granuloma. A thin, linear diffusion hypersignal was present at the lateral part of the attic.

- Case 5: a 7 years old boy. The diffusion hypersignal was very small: $3.5 \mathrm{~mm}$ in its largest diameter. The corresponding hyperdensity on the CT scan had an atypical bony margin. Regarding the pathological study, bony and cartilaginous tissues were found, without keratin nor squamous epithelium. 
In conclusion, two patients didn't have any ossicular lysis, three patients had T1 hypersignal, and two patients had a very small or thin DW-MRI hypersignal.

\section{DISCUSSION}

\section{Key Findings}

The number of DW-MRI of petrous bone for identification of cholesteatoma has grown since 2005. In our series, the observed PPV of $89 \%$ is certainly linked to the good detection of keratin. DWI sequences were DWI-TSE sequences known to minimize distorsion effects compared to echo planar diffusion (EPI-DW).

\section{Comparisons with other studies}

PPV of DW-MRI is around 96,3\% in children and 88,5\% in adults (1). Our results are consistent with the ones reported in the meta-analysis led by Van Egmond et al. demonstrating a better PPV in postoperative cases than in primary cases (2).

These PPV are a lot stronger than the ones calculated on CT scan images, which is around $67 \%$ (3). CT scan is usually systematic before the first surgery in order to study the petrous bone anatomy. The CT scan could be considered as enough before the first surgery, given that ossicular erosion corresponds to a cholesteatoma diagnosis in $90 \%$ of cases (4). However, for follow-up, MRI is preferred, due to the absence of ionizing radiation.

In our series, the use of DWI TSE sequences has led to a high positive predictive value of $89 \%$. DW-MRI hypersignal may artefactual du to T2-shine through phenomenon and thus, ADC map has to be considered. DW-MRI sequence is based on the Brownian motion of water molecules (5). The differential diagnoses of cholesteatoma in DW-MRI are abscess, bone powder, cholesterol granuloma, fat tissue, silicone sheet, wax, proteinaceous fluid, cartilage, tympanosclerosis and squamous cell carcinoma (6). Moreover, other artefacts can also produce false positives: dental braces, eddy current, susceptibility, ghosting, chemical shift or motion (7).

The size of the DW-MRI hypersignal seems to be an important element in the diagnosis. In our study, false positives have a smaller average size than true positives. The inferior limit of detection of the cholesteatoma is between 3 and $5 \mathrm{~mm}(7,8)$. Although there is not a precise cut-off, we should be very careful in case of a small DW-MRI hypersignal in order to avoid an unnecessary surgery. One might to choose to perform a complementary MRI 6 months later to assess the changes and eventually propose a surgery if the mass has increased in size and the signal is in favor of a cholesteatoma.

\section{Strengths and limitation of the study}

This is a strictly pediatric series. The main limitation of this study is the small number of false positives, which weakens the analysis of this group and the comparison with true positives. The PPV found in our study is consistent to the ones described in the literature as in adults as in children $(9,10)$. Moreover, we performed a critical analysis of our pitfalls (T1 hypersignal, size of the DW-MRI hypersignal), in order to later avoid unnecessary surgery.

\section{Clinical implications}

On MRI imaging, cholesteatoma shows a T2 hyposignal, DW-MRI hypersignal, and no T1 hypersignal. However, a DW-MRI hypersignal is not synonymous of cholesteatoma diagnosis. Indeed, this diagnosis relies on the importance of a proper otoscopic examination, a suggestive medical history, CT scan data and analysis of other MRI sequences, including T1-weighted sequence, to rule out other etiologies of middle ear DW-MRI hypersignal.

Despite these false positives, DW-MRI is a key element for cholesteatoma diagnosis which does not require contrast-enhanced MRI. Indeed, this may be an important element to consider since gadolinium can lead to nephrogenic systemic fibrosis and brain tissue retention; Gadolinium tends to deposit cumulatively in the brain of patients with normal hepatobiliary function and intact blood-brain barrier. This has to be put into 
perspective given that these children may have to perform repetitive MRI scans to detect any cholesteatoma recurrence.

DW-MRI duration is about 20 minutes. The average age of our patients was $14.2 \pm 5.3$ years old. They are consequently old enough and therefore do not require a general anesthesia to obtain a satisfactory exam quality. However, if the MRI exam has to be performed under general anesthesia, a second look surgery may then be preferable, since surgery is considered as the gold standard and it allows retrieval of the cholesteatoma.

MRI is very effective, especially for detecting cholesteatomas' recurrence and can be easily carried out since it does not require gadolinium injection nor sedation in older patients.

\section{BIBLIOGRAPHY}

1. Nash R, Wong PY, Kalan A, Lingam RK, Singh A. Comparing diffusion weighted MRI in the detection of post-operative middle ear cholesteatoma in children and adults. International Journal of Pediatric Otorhinolaryngology. 1 déc 2015;79(12):2281-5.

2. van Egmond SL, Stegeman I, Grolman W, Aarts MCJ. A Systematic Review of Non-Echo Planar DiffusionWeighted Magnetic Resonance Imaging for Detection of Primary and Postoperative Cholesteatoma. Otolaryngol Head Neck Surg. 1 fevr 2016;154(2):233-40.

3. Yiğiter AC, Pınar E, İmre A, Erdoğan N. Value of Echo-Planar Diffusion-Weighted Magnetic Resonance Imaging for Detecting Tympanomastoid Cholesteatoma. J Int Adv Otol. avr 2015;11(1):53-7.

4. Lemmerling MM, Foer BD, VandeVyver V, Vercruysse J-P, Verstraete KL. Imaging of the opacified middle ear. European Journal of Radiology. 1 juin 2008;66(3):363-71.

5. Bammer R. Basic principles of diffusion-weighted imaging. European Journal of Radiology. 1 mars 2003;45(3):169-84.

6. Veillon F, Collectif, Harmsworth VJ, Becker M. Imagerie de l'oreille et de l'os temporal : Tome 4, Tumeurs, nerf facial. Paris: Médecine Sciences Publications; 2013. 230 p.

7. Lingam RK, Nash R, Majithia A, Kalan A, Singh A. Non-echoplanar diffusion weighted imaging in the detection of post-operative middle ear cholesteatoma: navigating beyond the pitfalls to find the pearl. Insights Imaging. 24 août 2016;7(5):669-78.

8. Henninger B, Kremser C. Diffusion weighted imaging for the detection and evaluation of cholesteatoma. World J Radiol. 28 mai 2017;9(5):217-22.

9. Li PMMC, Linos E, Gurgel RK, Fischbein NJ, Blevins NH. Evaluating the utility of non-echo-planar diffusion-weighted imaging in the preoperative evaluation of cholesteatoma: A meta-analysis. The Laryngoscope. 1 mai 2013;123(5):1247-50.

10. Dubrulle F, Souillard R, Chechin D, Vaneecloo FM, Desaulty A, Vincent C. Diffusion-weighted MR imaging sequence in the detection of postoperative recurrent cholesteatoma. Radiology. fevr 2006;238(2):60410.

\section{Hosted file}

Table 1 20200408. docx available at https://authorea.com/users/310073/articles/440883-which-positivepredictive-value-for-diffusion-weighted-magnetic-resonance-imaging-for-children-s-cholesteatomasa-retrospective-study-of-126-mri-exams

\section{Hosted file}

Figure 1 20200408.docx available at https://authorea.com/users/310073/articles/440883-whichpositive-predictive-value-for-diffusion-weighted-magnetic-resonance-imaging-for-childrens-cholesteatomas-a-retrospective-study-of-126-mri-exams 